\title{
Detection and Local Staging of Prostate Cancer by 68Ga-PSMA-PET/CT, Comparison with mpMRI and Histopathology
}

\author{
F. Intema1, A. Kooistra' ${ }^{2}$, M. C. Vermeulen', R. M. Hoeben'1, A. M. van de Berk' ${ }^{1}$, A. Lont ${ }^{2}$, \\ W. Dingemans ${ }^{3}$, L. M. Andrews ${ }^{4}$, G. K. Lammers ${ }^{1}$, J. M. H. de Klerk ${ }^{1,2}$ \\ ${ }^{1}$ Department of Radiology and Nuclear Medicine, Meander Medical Centre, Amersfoort, The Netherlands \\ ${ }^{2}$ Department of Urology, Meander Medical Centre, Amersfoort, The Netherlands \\ ${ }^{3}$ Department of Pathology, Meander Medical Centre, Amersfoort, The Netherlands \\ ${ }^{4}$ Hospital Pharmacy, Meander Medical Centre, Amersfoort, The Netherlands \\ Email:jmh.de.klerk@meandermc.nl
}

How to cite this paper: Intema, F., Kooistra, A., Vermeulen, M.C., Hoeben, R.M., van de Berk, A.M., Lont, A., Dingemans, W., Andrews, L.M., Lammers, G.K. and de Klerk, J.M.H. (2020) Detection and Local Staging of Prostate Cancer by 68Ga-PSMAPET/CT, Comparison with mpMRI and Histopathology. Advances in Molecular Imaging, 10, 15-29.

https://doi.org/10.4236/ami.2020.103003

Received: June 30, 2020

Accepted: July 27, 2020

Published: July 30, 2020

Copyright (c) 2020 by author(s) and Scientific Research Publishing Inc. This work is licensed under the Creative Commons Attribution International License (CC BY 4.0).

http://creativecommons.org/licenses/by/4.0/

\section{(c) (i) Open Access}

\begin{abstract}
Introduction: 68Ga-PSMA-PET/CT has proven its value in prostate cancer with high positive predictive value for lymph node metastasis and superior detection of distant metastasis. There is growing evidence that 68Ga-PSMAPET/CT has high sensitivity for detection of tumor lesions in the prostate as well. Studies thus far have mainly been performed in patients prior to prostatectomy. Aim of this study is to evaluate diagnostic accuracy in a mixed population of men with increased risk of prostate cancer and evaluate diagnostic possibilities with respect to extra-capsular extension and seminal vesicle invasion. Methods: The population consisted of a retrospectively included sequential cohort of 69 patients with 68Ga-PSMA-PET/CT and mpMRI available. $68 \mathrm{Ga}-\mathrm{PSMA}-\mathrm{PET} / \mathrm{CT}$ was re-evaluated by two readers blinded for mPMRI and clinical information. Likelihood of tumour presence, extra-prostatic extension and seminal vesicle invasion was scored on 5-point Likert scale and localized schematically. Results were compared with mpMRI. Available pathological outcome served as gold standard. SUVmax of index lesions was measured and correlated to index tumor Gleason grade. Results: Clinically significant prostate cancer (Gleason $\geq 3+4$ ) was detected in 57 (83\%) of 69 patients. Diagnostic accuracy was $89 \%$ for PET reader $1,93 \%$ for PET reader 2 and $86 \%$ for mpMRI. Lesion concordance of 68Ga-PSMA-PET/CT and mpMRI was $97 \%$. SUVmax of the index lesion correlated to Gleason grade. Sensitivity for extracapsular extension in the prostatectomy group was $62 \%$
\end{abstract}


for PET reader 1, 33\% for PET reader 2 and 50\% for mpMRI. Specificity was $62 \%$ for PET reader $1,100 \%$ for PET reader 2 and $69 \%$ for mpMRI. Conclusion: Ga68-PSMA-PET shows high accuracy in the detection of tumor lesions in the prostate. Results on evaluating extra-capsular extension and seminal vesicle invasion are comparable to mpMRI. This study adds to the increasing evidence that $68 \mathrm{Ga}-\mathrm{PSMA}-\mathrm{PET} / \mathrm{CT}$ is imperative in detection of prostate cancer prior to biopsy.

\section{Keywords}

Prostate Cancer, Detection, Local Staging, 68-Gallium-Prostate Specific Membrane Antigen-Positron Emission Tomography, Multiparametric Magnetic Resonance Imaging

\section{Introduction}

Prostate cancer is a disease with high incidence and often has an indolent course. Adequate early detection of more aggressive cancers is imperative when aiming for curation. Meanwhile preventing unnecessary invasive diagnostic methods is important as well. It is key to accurately detect the location of those lesions with a high grade of malignancy. In patients with increased risk for prostate cancer based on raised serum level of prostate specific antigen (PSA), standard transrectal ultrasound (TRUS) guided biopsies may not be representative and malignancy grade can be underestimated. Several studies have confirmed the additional value of multi parametric magnetic resonance imaging (mpMRI) guided biopsies and recent guidelines support mpMRI-based targeted biopsies in biopsy naïve patients [1] [2]. mpMRI-guided biopsy while omitting random biopsies will be at cost of missing 5\% - 10\% of significant cancer [3] [4]. Sensitivity of mpMRI is lowest for lesions located ventrally in the prostate in the transition zone. This region is more difficult to evaluate on MRI than the peripheral zone and is traditionally not part of standardized random biopsies [5] [6]. Additionally, mpMRI positive lesions (PIRADS 4 or 5) lack specificity and are hard to differentiate from focal prostatitis, atrophy, scarring and benign hyperplasia nodules, resulting in a negative biopsy in $20 \%$ - $40 \%$ of cases [2] [7]. More important, accurate acquisition and evaluation of mpMRI is a critical challenge and demands experience and frequent exposure [8]. With the increased demand for mpMRI's for cancer detection, an alternative diagnostic tool with straightforward accurate detection would be welcome. Moreover, an alternative to mpMRI would also be a solution for patients ineligible for an MRI because of metal implants or claustrophobia.

PSMA-PET/CT was initially indicated for detection of location of tumor activity in patients with biochemical recurrence [9]. High diagnostic accuracy for metastatic disease led to PSMA-PET/CT being advised for primary staging in 
patients with high risk and intermediate risk cancers as an alternative for the total body bone scan or 18F-Methylcholine-PET [10]. Recent studies have shown promising positive predictive values in the detection of lymph node metastasis [11] [12] [13]. In addition to regional and distant staging, a logical next step would be to determine the role of PSMA PET/CT in local detection and staging (T-stage) of prostate cancer.

Several studies have shown beneficial results for the PSMA-PET/CT compared to $\mathrm{mpMRI}$ in primary tumor detection in a high risk population with proven prostate cancer [14] [15] [16] [17] [18]. Better delineation of tumor volume has been shown which could be relevant for planning of radiation therapy [19]. Lopci et al. showed high sensitivity and specificity in PSMA-PET/TRUS fused biopsies for detection of prostate cancer [20]. Hoffmann et al. concluded that 68GaPSMA-PET/CT guided biopsies increased diagnostic precision and reduced detection of insignificant cancers [21]. PSMA-receptor expression is correlated to malignancy grade which is important for obtaining representative biopsies [22]. Besides Ga68-PSMA, there are other PSMA bound tracers: a study of Turkbey et al. with $18 \mathrm{~F}-\mathrm{DCFBC}-\mathrm{PET} / \mathrm{CT}$ showed a sensitivity of only $61 \%$ while mpMRI performed really well (92\%) [23]. A study with 18F-PSMA-1007-PET/CT, showed good diagnostic accuracy compared to mpMRI (75\% and $73 \%$ respectively) [24].

Von Klot et al. found promising results regarding the ability of PSMA scan to determine lobe infiltration, extra-capsular extension (ECE) and seminal vesicle invasion (SVI) [25]. Muehlematter et al. reported increased sensitivity for ECE and SVI in favor of Ga68-PSMA-PET/MR in comparison with mpMRI at cost of a slight decrease in specificity [26].

The majority of studies thus far have been performed in patients with proven prostate cancer prior to prostatectomy. In these populations, sensitivity could be overestimated because of the awareness of the readers that tumor was present. Specificity on a per patient base cannot be determined when the whole included population has disease presence. It would be of interest to compare 68Ga-PSMA$\mathrm{PET} / \mathrm{CT}$ to $\mathrm{mpMRI}$ regarding local detection and staging in a clinical setting with a population with uncertainty regarding the presence and extension of prostate cancer. When results for 68Ga-PSMA-PET/CT prove to be comparable to mpMRI, one might even consider omitting mpMRI in de primary work-up in the future. In intermediate and high risk patients there is already an indication for 68GA-PSMA PET/CT for regional and distant staging. Evaluation of 68GaPSMA-PET/CT in studies which aim at determining accuracy, should be blinded for mpMRI results. Otherwise, results will not be representative for clinical use when diagnosis prostate cancer is not yet been established.

Primary objective of this study is to compare 68Ga-PSMA-PET/CT to mpMRI with respect to detection and localization of the primary tumor and in defining local T-stagein a mixed population with and without clinically significant prostate cancer. 


\section{Patients and Methods}

\subsection{Study Population}

The study was designed as a comparative study between the 68Ga-PSMA-PET/CT and mpMRI prostate performed for primary detection and staging in a retrospectively included cohort. Study population consisted of allpatients in whom a 68Ga-PSMA-PET/CT was performed between October 2016 and March 2019 and who were treated in the Meander Medical Centre Amersfoort. Ethical approval of the hospital board was given. The study was conducted according to the principles of the Declaration of Helsinki [27] and in accordance with the Medical Research Involving Human Subjects Act [28].

Inclusion criteria were availability of mpMRI prostate and 68Ga-PSMAPET/CT obtained within a one year interval. Patients who already underwent treatment for prostate cancer before either the mPMRI or 68Ga-PSMA-PET/CT was performed were excluded. One patient showed bladder cancer after revision of biopsy histopathology and was excluded. 69 patients were available for analysis. In 68 patients, biopsy histopathology results of the prostate were available, one diagnosis was made based on a histopathological proven lymph node metastasis. In 31 patients prostatectomy was performed with additional definitive histopathological results available of the entire prostate and extra-prostatic extend.

\subsection{Imaging Techniques}

PET/CT (Siemens PET/CT Biograph mCT S40) from head to upper legs (3 minutes per table position) and non-contrast enhanced CT (regular dose) for attenuation correction and anatomical correlation was performed 60 minutes $( \pm 5)$ after intravenous administration of $1.5 \mathrm{MBq}$ per kg body-weight 68Ga PSMAHBED-CC, maximum dosage $187.5 \mathrm{MBq}$ and $10 \mathrm{mg}$ furosemide. 68Ga-PSMA was produced on-site according to good manufactory practices using a Ge68Ga68 generator (Isotope Technologies Garching; ITG, Munchen, Germany). Two readers (R1 and R2) re-evaluated all 68Ga-PSMA-PETs blinded for clinical information and mpMRI images using a standardized protocol. Presence of a lesion was determined as increased focal activity related to prostate background. Likelihood of the lesion being clinically significant (Gleason $\geq 3+4$ ) cancer was scored on a Likert scale from 1 - 5 (1; not present, 2; unlikely, 3; possibly, 4; likely, 5 very likely). Most active (index) lesions (lesion defined as PI-RADS $\geq 3+3$ for mpMRI and Likert 3 for 68Ga-PSMA-PET) were localized schematically in a 12-segment model [16]. Standardized uptake value (maximum) of the most active region was registered as well as SUVmean of background prostate by central placement of a VOI of $1 \mathrm{~cm}^{3}$, with caution not to include a lesion. Suspected extra-capsular extension was scored on a Likert scale $(1-5)$ based on proximity to, or extension beyond prostate capsule of activity maximum. Suspected seminal vesicle invasion was evaluated similarly, based on lesion proximity to base of 
seminal vesicles and on focal intensity.

MRI was performed on a Philips Achieva 1.5T after intravenous administration of $20 \mathrm{mg}$ Buscopan. T1w, T2w, diffusion 0-50-400-800-1200 and dynamic contrast enhanced series after administration of $20 \mathrm{cc}$ Gadolinium, $2.5 \mathrm{cc} / \mathrm{sec}$ were obtained. Evaluation was performed in clinical setting by one of two experienced radiologists according to PI-RADS-v2 protocol. Localization in the 12segment-model was done additionally for study purposes.

\subsection{Histological Examination}

Random biopsy was performed by TRUS, at least 4 cores left and right were acquired, withadditional cores depending on prostate size. In case of palpable or hypo-echogenic lesions, additional targeted biopsy was performed. mpMRI guided biopsies were performed in nine patients because of negative random biopsies and persistent suspicion of prostate cancer. Histopathological evaluation was done according to ISUP standard protocols for Gleason grading by experienced pathologists [29]. Clinically significant disease was defined as Gleason $3+4$ of higher. In a subgroup of patient with prostatectomy, presence of extra-capsular extension and seminal vesicle invasion was registered. In case of discrepancy between results of biopsies and prostatectomy, results of the latter overruled earlier results.

\subsection{Assessing Imaging and Histopathological Concordance}

68Ga-PSMA-PET/CT was considered positive for lesions with Likert 4 and 5 scores. mpMRI was positive in case of PI-RADS 4 and 5 scores. Histopathological Gleasongrade $\geq 3+4$ was considered as clinically significant prostate cancer.

Concordance of lesionlocation was defined as presence of a lesion (PI-RADS or Likert $\geq 3$ ) in a least one corresponding segment. Discordance was defined as presence of a lesion (optionally a second lesion) with no identical segments (Figure 1). Lesion absence in both 68Ga-PSMA-PET/CT and mpMRI was considered as concordance. Tumor volume on 68Ga-PSMA-PET/CT was compared to $\mathrm{mPMRI}$ by per patients registration whether more segment were involved on 68Ga-PSMA-PET/CT or on mpMRI (Figure 1).

\subsection{Statistical Analysis}

A quantitative description (continuous or ordinal scale) of different parameters was given (median or mean and standard deviation). Comparison of the sensitivity, specificity, positive and negative predictive values as well as accuracy of the diagnostic test was presented with histopathology as gold standard. Extracapsular extension and seminal vesicle invasion was compared to mpMRI for the whole group and an additional analysis was performed in a subgroup of patients that had a prostatectomy.

Correlation between SUV max and Gleason grade was determined by twosided spearman rho with correlation coefficient and p-value presented. Location 

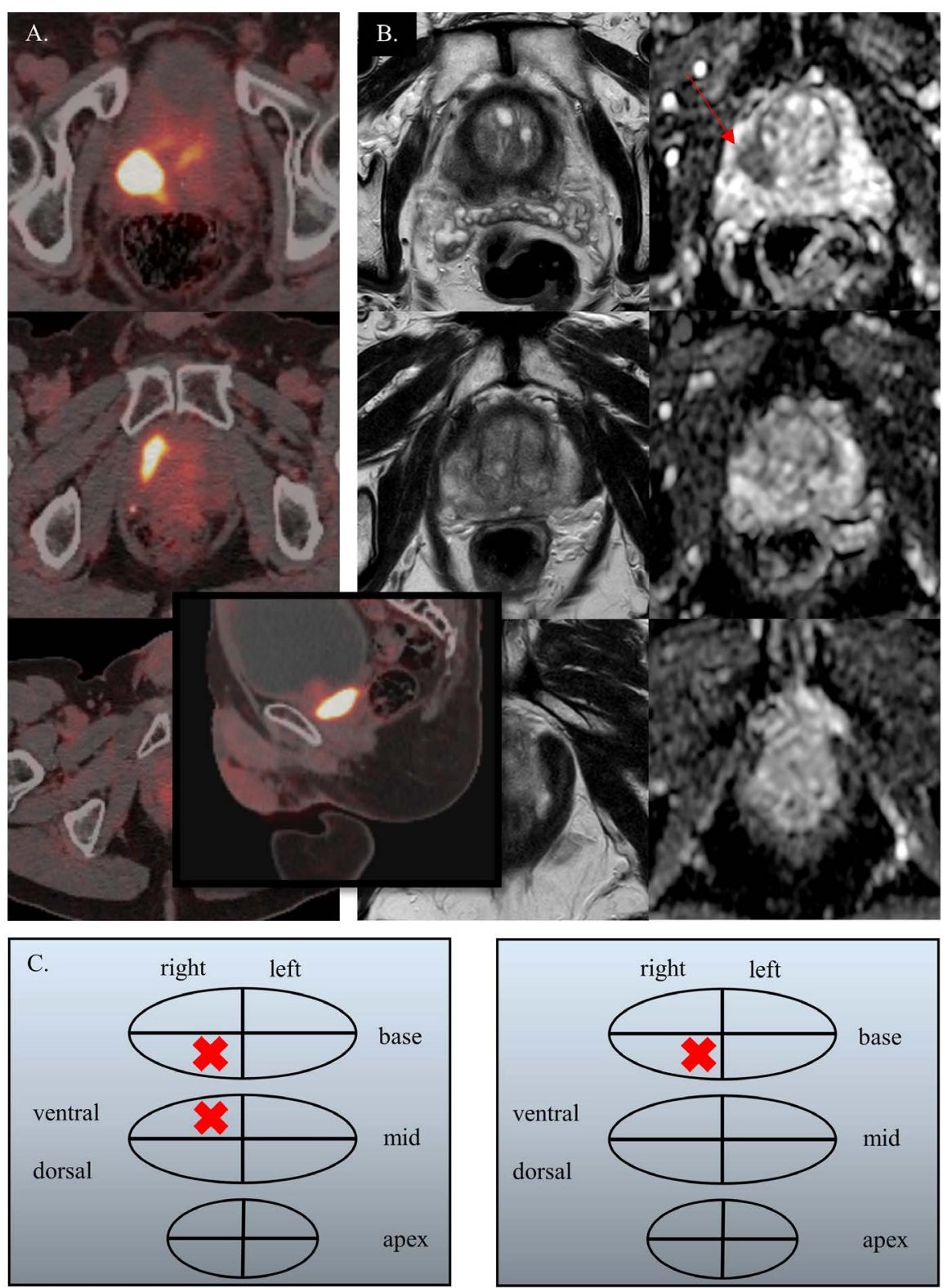

Figure 1. Comparison of index lesions on 68Ga-PSMA-PET/CT (A) and mpMRI (B). Lesion location was registered in a 12 -segment model (C). Lesion size on PET and mpMRI was compared by number of involved segments per lesion with corresponding segments. When there was no corresponding segment, it was interpreted as separate lesion. In this example, lesion location is concordant and lesion size is larger on 68Ga-PSMA-PET. Sagittal view (inset) confirms one lesion on 68Ga-PSMA-PET/CT and not two separate ones.

concordance was set as a per-patient categorical variable (all matching locations: yes or no).

\section{Results}

Sixty-nine patients were included, characteristics are shown in Table 1. Mean time between mpMRI and 68Ga-PSMA-PET/CT was forty days (SD \pm 106 ). 
Table 1. Patient characteristics. Risk stratification based on iPSA, gleason values and clinical T-stage (digital rectal exam). ADT (androgen deprivation therapy) EBRT (external beam radiation therapy) WW (watchfull waiting).

\begin{tabular}{ccc}
\hline Age & $67 \pm 7$ (mean \pm SD) & \\
\hline PSA ng/l (median) & 17.0 (median) & $3.8-120$ (range) \\
\hline $\begin{array}{c}\text { Clinically significant prostatecancer } \\
\text { Pre-treatment risk stratification } \\
\text { for metastatic disease }\end{array}$ & Gleasongrade $\geq 3+4$ & $57(81)$ \\
\hline $\begin{array}{c}\text { Suspectedmetastasis } \\
\text { (PSMA-PET) }\end{array}$ & Low & $5(7)$ \\
\hline & Intermediate & $17(25)$ \\
& High & $47(68)$ \\
\hline Treatment & Distant (M1a/b/c) & $18(26)$ \\
& RALP & $11(16)$ \\
\hline & EBRT & $31(45)$ \\
& ADT (including postponed) & $11(16)$ \\
& -with or without chemo- or radiotherapy & $18(26)$ \\
& Active surveillance/WW & $9(13)$
\end{tabular}

Clinically significant prostate cancer (Gleason $\geq 3+4$ ) was detected in $57(83 \%)$ of 69 patients, with one diagnosis based on lymph node biopsy. Six patients had a negative biopsy and six showed Gleason $3+3$ cancer. 68Ga-PSMA-PET/CT reader 1 (R1) showed false negative results in three patients with Gleason $\geq 3+4$ and reader 2 (R2) showed no false negative results. mpMRI showed false negative results in five patients (Table 2). This resulted in a sensitivity of $95 \%$ for R1, $100 \%$ for R2 and 91\% for mpMRI. Specificity for Gleason $\geq 3+4$ was $67 \%$ for both PET readers and for mpMRI as well. Negative predictive valuewas $73 \%$ for R1, $100 \%$ for R2 and 62\% mpMRI. Positive predictive value was $93 \%$ for all. Diagnostic accuracy was $89 \%$ for R1, 93\% for R2 and $86 \%$ for mpMRI.

In 60 patients a lesion (PIRADS $\geq 3$ ) was present on mpMRI. In 58 patients (97\%) a concordant lesion was registered on 68Ga-PSMA-PET/CT (Likert $\geq 3$ ). In $46 \%$ of patients more segments were involved on $68 \mathrm{Ga}-\mathrm{PSMA}-\mathrm{PET} / \mathrm{CT}$ versus in $23 \%$ of patients on mpMRI.

SUVmax of the most active lesion was correlated to Gleason grade (Figure 2). SUVmean background had a range of 1.0 to 3.0. The correlation of SUVmax with Gleason grade was slightly better than the correlation of the ratio of SUVmax target lesion/SUV mean background with Gleason grade, $r=0.583$ vs $r=$ 0.518 . Lowest SUVmax value for a clinically significant lesion (Gleason $\geq 3+4$ ) was 4.2. Highest value for a lesion in a patient without prostate cancer was 4.8. Two patients with Gleason $3+3$ prostate cancer had a SUVmax of 15.1 and 16 .

Extracapsular extension (ECE) was scored positive on 68Ga-PSMA-PET/CT in 37 patients by reader 1 and in 19 patients by reader 2, mpMRI scored 30 of 69 
Table 2. Diagnostic results of MRI and PET (R1; reader 1, R2; reader 2) compared to histopathology results. A positive result was defined as PI-RADS $\geq 4$ or Likert $\geq 4$. False positive results for MRI and two PET readers were comparable. False negative results were more present in MRI (including missing of 2 Gleason 8 lesions). ${ }^{\star}$ histopathology from lymph node metastasis, no Gleason grade available.

\begin{tabular}{cccccccc}
\hline \multicolumn{2}{c}{ Pathology } & \multicolumn{2}{c}{ MRI $(\mathrm{n}=)$} & \multicolumn{2}{c}{ PET R1 $(\mathrm{n}=)$} & \multicolumn{2}{c}{ PET R2 $(\mathrm{n}=)$} \\
\hline Gleason & $(\mathrm{n}=)$ & Positive & Negative & Positive & Negative & Positive & Negative \\
\hline benign & 6 & 1 & 5 & 2 & 4 & 1 & 5 \\
6 & 6 & 3 & 3 & 2 & 4 & 3 & 3 \\
7 & 30 & 27 & 3 & 27 & 3 & 30 & 0 \\
8 & 12 & 10 & 2 & 12 & 0 & 12 & 0 \\
9 & 10 & 10 & 0 & 10 & 0 & 10 & 0 \\
10 & 4 & 4 & 0 & 4 & 0 & 4 & 0 \\
Metastasis $^{\star}$ & 1 & 1 & 0 & 1 & 0 & 1 & 0 \\
\hline
\end{tabular}

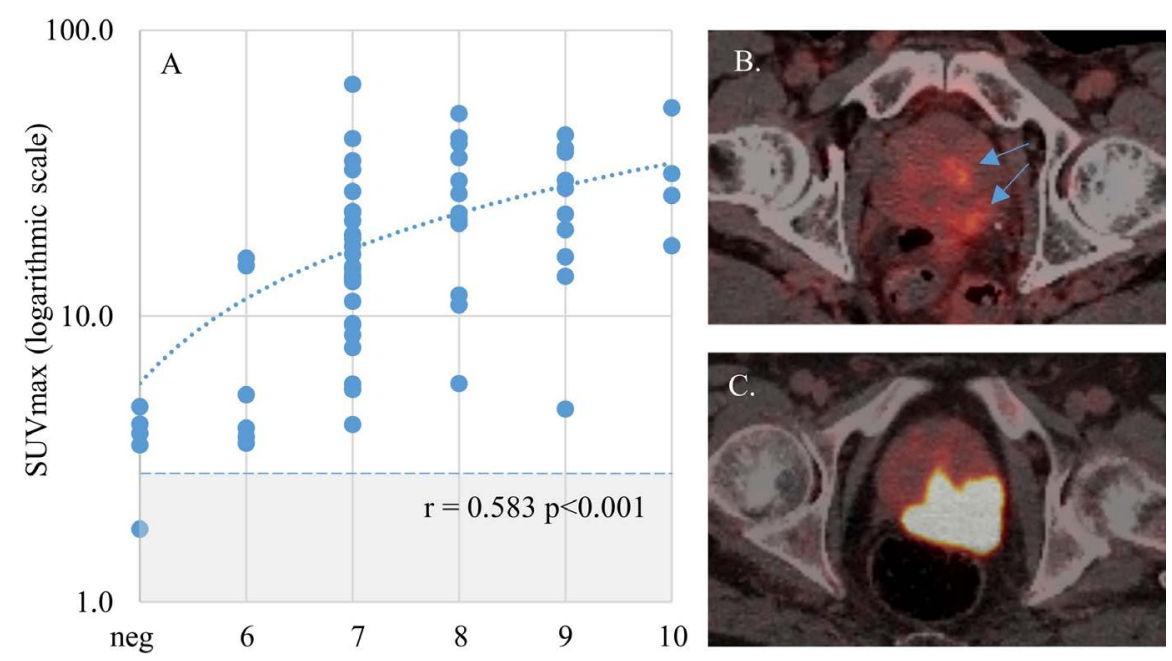

Figure 2. (A) Correlation between maximal prostate activity (SUVmax in prostate) and Gleason grade. Mean (SUVmean) background prostate activity was $2.0 \pm 0.5$ (range 1.0 3.0; dotted line). (B) example of Gleason $3+3$ tumor (arrows) with low PSMA-binding (SUVmax 4.1) (C) example of Gleason $5+4$ tumor with high PSMA-binding (SUVmax 37.4) and extra-prostatic extension.

patients as positive. Diagnosis in 68Ga-PSMA-PET/CT and mpMRI was consistent in $63 \%$ of patients for both readers. Seminal vesicle invasion (SVI) was present on 68Ga-PSMA-PET/CT in 10 patient for R1 and in 15 patients for R2, mpMRI scored positive in 14 of 69 patients. Diagnosis in 68Ga-PSMA-PET/CT was consistent with mpMRI in $90 \%$ of patients for reader 1 and $89 \%$ for reader 2 .

Prostatectomy was performed in 31 of 69 patients. 18 of those patients had ECE and six SVI. In two patients both 68Ga-PSMA-PET/CT and mpMRI scored SVI as positive. The others were falsely negative by interpreting Likert 3 as negative (Figure 3). Results of evaluation of ECE and SVI are presented in Table 3. 
Table 3. Diagnostic results of MRI and 68Ga-PSMA-PET/CT (R1; reader 1, R2; reader 2) compared to histopathology results. A positive result was defined as Likert $\geq 4$.

\begin{tabular}{|c|c|c|c|c|c|}
\hline & \multicolumn{5}{|c|}{ Prostatectomy subgroup $(\mathrm{n}=31)$} \\
\hline \multirow{7}{*}{$\begin{array}{c}\text { Extracapsular } \\
\text { extension }\end{array}$} & & & R1 & R2 & mpMRI \\
\hline & \multirow{2}{*}{ Histopathology } & Positive (n) & $11 / 18$ & $6 / 18$ & $9 / 18$ \\
\hline & & Negative (n) & $8 / 13$ & $13 / 13$ & $9 / 13$ \\
\hline & Sensitivity & & $61 \%$ & $33 \%$ & $50 \%$ \\
\hline & Specificity & & $62 \%$ & $100 \%$ & $69 \%$ \\
\hline & Positive predictive value & & $69 \%$ & $100 \%$ & $69 \%$ \\
\hline & \multicolumn{2}{|l|}{ Negative predictive value } & $53 \%$ & $52 \%$ & $50 \%$ \\
\hline \multirow{7}{*}{$\begin{array}{l}\text { Seminal vesicle } \\
\text { invasion }\end{array}$} & & & $\mathrm{R} 1$ & R2 & mpMRI \\
\hline & \multirow{2}{*}{ Histopathology } & Positive (n) & $2 / 6$ & $2 / 6$ & $2 / 6$ \\
\hline & & Negative (n) & $25 / 25$ & $25 / 25$ & $25 / 25$ \\
\hline & Sensitivity & & $33 \%$ & $33 \%$ & $33 \%$ \\
\hline & Specificity & & $100 \%$ & $100 \%$ & $100 \%$ \\
\hline & Positive predictive value & & $100 \%$ & $100 \%$ & $100 \%$ \\
\hline & Negative predictive value & & $86 \%$ & $86 \%$ & $86 \%$ \\
\hline
\end{tabular}
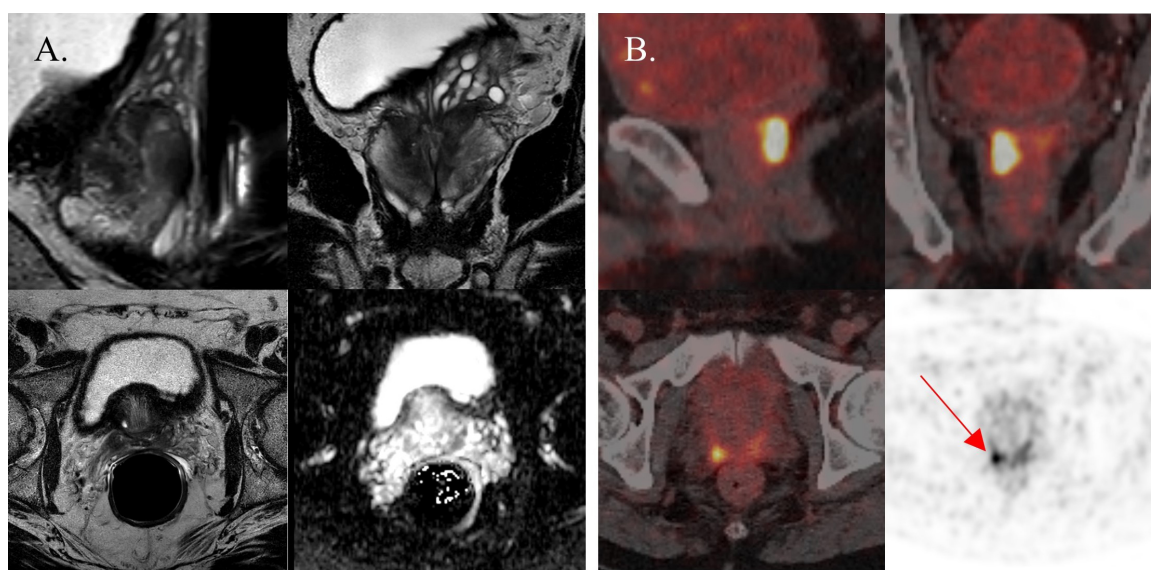

Figure 3. Evaluation of seminal vesicle invasion. (A) mpMRI: symmetrical base of seminal vesicles on T2 weighted images without diffusion restriction ((A) lower right panel), invasion not likely. (B) Asymmetrical uptake of PSMA (arrow) with increase in the base of right seminal vesicle, suspect for T3b stage. Histopathology showed invasion of the base of the right seminal vesicle.

\section{Discussion}

68Ga-PSMA-PET/CT detected more patients with clinically significant prostate cancer than mpMRI. Sensitivity for clinically significant cancer was slightly higher (reader $195 \%$ and reader 2 100\%) for 68Ga-PSMA-PET/CT than for mpMRI (91\%) with equal specificity. These results are in line with previous published studies. Kalapara et al. showed a sensitivity of $94 \%$ for both $68 \mathrm{Ga}-\mathrm{PSMA}-\mathrm{PET} / \mathrm{CT}$ 
and mpMRI [18], and Donato et al. showed a sensitivity of $94 \%$ for $68 \mathrm{Ga}-$ PSMA-PET/CT with lower detection rate for mPMRI [14]. Specificity in our study was $67 \%$, this is lower than in the study of Donato et al. (90\%) and could be dependent on population composition. The chance of a false positive lesion is lower in a population of men with proven prostate cancer, than in a mixed population composed of both men with prostate cancer and without. However, Lopci et al. showed high specificity of $90 \%$ in a mixed population as well [30]. They performed PSMA-guided biopsies. In our study, the histopathological gold standard was random biopsy and could have been falsely negative. For example, one patient with a very suspicious lesion on both 68Ga-PSMA-PET/CT and mpMRI had negative biopsy, two years later Gleason $4+4$ prostate cancer was detected. This case emphasises the importance of image guided biopsy.

Locations on mpMRI and 68Ga-PSMA-PET/CT were concordant, without more additional foci on PET. Donato et al. showed beneficial results for $68 \mathrm{Ga}$ PSMA-PET/CT to detect multifocal disease, which implied a higher sensitivity for 68Ga-PSMA-PET/CT. Two other studies showed larger tumour volumes on 68Ga-PSMA-PET/CT [15] [31]. This is in line with our results, as 68Ga-PSMA$\mathrm{PET} / \mathrm{CT}$ showed more affected segments.

Earlier studies showed that approximately $5 \%-10 \%$ of clinically significant prostate cancer does not show high PSMA-receptor expression [32]. Our population included a patient with a Gleason $5+4$ tumour with relatively low PSMAuptake. Despite low uptake, this tumour was indicated as target lesion by standing out against prostate background activity. Uprimny et al. showed that in $9 \%$ of patients the lesion did not stand out from prostate background [22]. These were all Gleason 6 or 7 graded tumours and PSA levels were $<10 \mathrm{ng} / \mathrm{ml}$. PSA levels of the three falsely negative patients in our study as evaluated by reader 1 on 68Ga-PSMA-PET/CT were $8.8 ; 3.9$ and $8.9 \mathrm{ng} / \mathrm{ml}$.

Sensitivity of ECE was low for both 68Ga-PSMA-PET/CT and mpMRI. Sensitivity for ECE with mpMRI in our study was only $50 \%$, comparable with the acknowledged low sensitivity $57 \%$ in a meta-analysis [33]. However, specificity in this meta-analysis was high (91\%), while our specificity was $69 \%$. Specificity for mpMRI may have been low because "likely" ECE was included as positive. There are a few studies that evaluated extra-prostatic extension on PSMA-PET/CT. Klot et al. showed high sensitivity of $90 \%$ based on 6 positive patients in a population of 21 [25]. Muehlematter et al. showed an increase in sensitivity from 46 to $69 \%$ when adding PSMA-PET to mpMRI [26]. In our study ECE was based on intensity of the lesion and proximity to the capsule. However, inter-reader differences were substantial. Acceptable inter-reader differences regarding sensitivity were decribed by Muehlematter et al. who included four readers, inter-reader reliability regarding specificity was better. More training would probably result in more coherent results of both readers. While Muehlematter et al. advocated for the combined values of PSMA-PET and mPMRI, Chen et al. showed that mpMRI has no additional value and Yilmaz shows no additional value of PSMA- 
PET for diagnosis ECE [34]. Criteria regarding ECE evaluation could be of influence, diagnosis based on angulated contour and obliteration of recto-prostatic angle showed best results [25] [34].

Whole group results in our study for diagnosis SVI in 68Ga-PSMA-PET/CT were more robust than for ECE and showed acceptable inter-reader variation. Diagnosis was consistent with mpMRI results. Sensitivity and specificity cannot be reliable determined as numbers in the prostatectomy-subgroup were too small. This problem is difficult to overcome as was emphasised by others as patients with SVI will often not have a prostatectomy and as such no definitive histopathology is available [25] [26]. Most mpMRI studies encountered this problem as well [33].

Study results show convincing evidence of additional value of 68Ga-PSMA$\mathrm{PET} / \mathrm{CT}$ in detection of prostate cancer. For successful clinical use, PET guided biopsies are required. Several have shown that PET/CT-TRUS fused biopsies are technical feasible and these experiences are shared by our group [20] [35] [36] [37].

The PROMIS-trial has shown that using mpMRI to triage men might allow $27 \%$ of patients avoid a primary biopsy and diagnosis of $5 \%$ fewer clinically insignificant cancers [1]. Sensitivity was 93\%. Results for PSMA-PET imply that sensitivity might even be better than with mpMRI. Donato et al. found clinically significant prostate cancer in $70 \%-80 \%$ of patient with negative mpMRI [17]. This could be at cost of finding more clinically insignificant cancer. Our study showed intense PSMA-uptake in two patients with Gleason $3+3$ tumour, mpMRI was evaluated as PI-RADS 3 and 4. Uprimny et al. showed comparable results with a positive correlation between SUVmax and Gleason grade, but with several outliers, SUVmax values for Gleason $3+3$ had a range up to 19.3 [22].

\section{Conclusion}

Blinded evaluation of 68Ga-PSMA-PET/CT showed higher sensitivity than mpMRI for the overall detection of primary prostate cancer in a clinical cohort of patients with and without prostate cancer. Evaluation of extra-prostatic extension and seminal vesicle invasion on 68Ga-PSMA-PET/CT is comparable to mpMRI. 68Ga-PSMA-PET/CT can probably replace mpMRI as initial diagnostic step, while PET-guided biopsies are feasible.

\section{Conflicts of Interest}

The authors declare no conflicts of interest regarding the publication of this paper.

\section{References}

[1] Ahmed, H.U., El-ShaterBosaily, A., Brown, L.C., Gabe, R., Kaplan, R., Parmar, M.K., et al. (2017) Diagnostic Accuracy of Multi-Parametric MRI and TRUS Biopsy in Prostate Cancer (PROMIS): A Paired Validating Confirmatory Study. The Lancet, 389, 815-822. https://doi.org/10.1016/S0140-6736(16)32401-1 
[2] Kasivisvanathan, V., Rannikko, A.S., Borghi, M., Panebianco, V., Mynderse, L.A., Vaarala, M.H., et al. (2018) MRI-Targeted or Standard Biopsy for Prostate-Cancer Diagnosis. The New England Journal of Medicine, 19, 1767-1777. https://doi.org/10.1056/NEJMoa1801993

[3] Dell'Oglio, P., Stabile, A., Soligo, M., Brembilla, G., Esposito, A., Gandaglia, G., et al. (2020) There Is No Way to Avoid Systematic Prostate Biopsies in Addition to Multiparametric Magnetic Resonance Imaging Targeted Biopsies. European Urology Oncology, 1, 112-118. https://doi.org/10.1016/j.euo.2019.03.002

[4] Kohestani, K., Wallstrom, J., Dehlfors, N., Sponga, O.M., Mansson, M., Josefsson, A., et al. (2019) Performance and Inter-Observer Variability of Prostate MRI (PIRADS Version 2) outside High-Volume Centres. Scandinavian Journal of Urology, 5, 304-311. https://doi.org/10.1080/21681805.2019.1675757

[5] Dianat, S.S., Carter, H.B. and Macura, K.J. (2014) Performance of Multiparametric Magnetic Resonance Imaging in the Evaluation and Management of Clinically LowRisk Prostate Cancer. Urologic Oncology, 1, 39.e1-10. https://doi.org/10.1016/j.urolonc.2013.04.002

[6] Schouten, M.G., van der Leest, M., Pokorny, M., Hoogenboom, M., Barentsz, J.O., Thompson, L.C. and Fütterer, J.J. (2016) Why and Where Do We Miss Significant Prostate Cancer with Multi-Parametric Magnetic Resonance Imaging Followed by Magnetic Resonance-Guided and Transrectal Ultrasound-Guided Biopsy in Biopsy-Naïve Men? European Urology, 6, 896-903. https://doi.org/10.1016/j.eururo.2016.12.006

[7] Woo, S., Suh, C.H., Kim, S.Y., Cho, J.Y. and Kim, S.H. (2017) Diagnostic Performance of Prostate Imaging Reporting and Data System Version 2 for Detection of Prostate Cancer: A Systematic Review and Diagnostic Meta-Analysis. European Urology, 2, 177-188. https://doi.org/10.1016/j.eururo.2017.01.042

[8] Purysko, A.S., Bittencourt, L.K., Bullen, J.A., Mostardeiro, T.R., Herts, B.R. and Klein, E.A. (2017) Accuracy and Interobserver Agreement for Prostate Imaging Reporting and Data System, Version 2, for the Characterization of Lesions Identified on Multiparametric MRI of the Prostate. American Journal of Roentgenology, 2, 339-349. https://doi.org/10.2214/AJR.16.17289

[9] Eissa, A., Elsherbiny, A., Coelho, R.F., Rassweiler, J., Davis, J.W., Porpiglia, F., et al. (2018) The Role of 68Ga-PSMA PET/CT Scan in Biochemical Recurrence after Primary Treatment for Prostate Cancer: A Systematic Review of the Literature. Minerva Urologica e Nefrologica, 5, 462-478. https://doi.org/10.23736/S0393-2249.18.03081-3

[10] Corfield, J., Perera, M., Bolton, D. and Lawrentschuk, N. (2018) (68)Ga-Prostate Specific Membrane Antigen (PSMA) Positron Emission Tomography (PET) for Primary Staging of High-Risk Prostate Cancer: A Systematic Review. World Journal of Urology, 4, 519-527. https://doi.org/10.1007/s00345-018-2182-1

[11] Kopp, J., Kopp, D., Bernhardt, E., Manka, L., Beck, A., Gerullis, H., et al. (2020) (68)Ga-PSMA PET/CT Based Primary Staging and Histological Correlation after Extended Pelvic Lymph Node Dissection at Radical Prostatectomy. World Journal of Urology. https://doi.org/10.1007/s00345-020-03131-0

[12] Luiting, H.B., van Leeuwen, P.J., Busstra, M.B., Brabander, T., van der Poel, H.G., Donswijk, M.L., et al. (2020) Use of Gallium-68 Prostate-Specific Membrane Antigen Positron-Emission Tomography for Detecting Lymph Node Metastases in Primary and Recurrent Prostate Cancer and Location of Recurrence after Radical Prostatectomy: An Overview of the Current Literature. BJU International, 2, 206-214. https://doi.org/10.1111/bju.14944 
[13] Maurer, T., Gschwend, J.E., Rauscher, I., Souvatzoglou, M., Haller, B., Weirich, G., et al. (2016) Diagnostic Efficacy of 68Gallium-PSMA Positron Emission Tomography Compared to Conventional Imaging for Lymph Node Staging of 130 Consecutive Patients with Intermediate to High Risk Prostate Cancer. The Journal of Urology, 5, 1436-1443. https://doi.org/10.1016/j.juro.2015.12.025

[14] Donato, P., Morton, A., Yaxley, J., Ranasinghe, S., Teloken, P.E., Kyle, S., et al. (2020) (68)Ga-PSMA PET/CT Better Characteriseslocalised Prostate Cancer after MRI and Transperineal Prostate Biopsy: Is (68)Ga-PSMA PET/CT Guided Biopsy the Future? European Journal of Nuclear Medicine and Molecular Imaging, 8, 18431851. https://doi.org/10.1007/s00259-019-04620-0

[15] Spohn, S., Jaegle, C., Fassbender, T.F., Sprave, T., Gkika, E., Nicolay, N.H., et al. (2020) Intraindividual Comparison between (68)Ga-PSMA-PET/CT and mpMRI for Intraprostatic Tumor Delineation in Patients with Primary Prostate Cancer: A Retrospective Analysis in 101 Patients. European Journal of Nuclear Medicine and Molecular Imaging. https://doi.org/10.1007/s00259-020-04827-6

[16] Scheltema, M.J., Chang, J.I., Stricker, P.D., van Leeuwen, P.J., Nguyen, Q.A., Ho, B., et al. (2019) Diagnostic Accuracy of (68)Ga-Prostate-Specific Membrane Antigen (PSMA) Positron-Emission Tomography (PET) and Multiparametric (mp) MRI to Detect Intermediate-Grade Intra-Prostatic Prostate Cancer Using Whole-Mount $\mathrm{Pa}$ thology: Impact of the Addition of (68)Ga-PSMA PET to mpMRI. BJU International, 124, 42-49. https://doi.org/10.1111/bju.14794

[17] Donato, P., Roberts, M.J., Morton, A., Kyle, S., Coughlin, G., Esler, R., et al. (2019) Improved Specificity with (68)Ga PSMA PET/CT to Detect Clinically Significant Lesions "Invisible" on Multiparametric MRI of the Prostate: A Single Institution Comparative Analysis with Radical Prostatectomy Histology. European Journal of Nuclear Medicine and Molecular Imaging, 1, 20-30.

https://doi.org/10.1007/s00259-018-4160-7

[18] Kalapara, A.A., Nzenza, T., Pan, H.Y., Ballok, Z., Ramdave, S., O’Sullivan, R., et al. (2019) Detection and Localisation of Primary Prostate Cancer Using (68)Ga-PSMA PET/CT Compared with mpMRI and Radical Prostatectomy Specimens. BJU International. https://doi.org/10.1111/bju.14858

[19] Bettermann, A.S., Zamboglou, C., Kiefer, S., Jilg, C.A., Spohn, S., Kranz-Rudolph, J., et al. (2019) (68)Ga-PSMA-11 PET/CT and Multiparametric MRI for Gross Tumor Volume Delineation in a Slice by Slice Analysis with Whole Mount Histopathology as a Reference Standard Implications for Focal Radiotherapy Planning in Primary Prostate Cancer. Radiotherapy and Oncology, 141, 214-219. https://doi.org/10.1016/j.radonc.2019.07.005

[20] Lopci, E., Saita, A., Lazzeri, M., Lughezzani, G., Colombo, P., Buffi, N.M., et al. (2018) (68)Ga-PSMA Positron Emission Tomography/Computerized Tomography for Primary Diagnosis of Prostate Cancer in Men with Contraindications to or Negative Multiparametric Magnetic Resonance Imaging: A Prospective Observational Study. Journal of Urology, 1, 95-103. https://doi.org/10.1016/j.juro.2018.01.079

[21] Hofman, M.S., Murphy, D.G., Williams, S.G., Nzenza, T., Herschtal, A., Lourenco, R.A., et al. (2018) A Prospective Randomized Multicentre Study of the Impact of Gallium-68 Prostate-Specific Membrane Antigen (PSMA) PET/CT Imaging for Staging High-Risk Prostate Cancer Prior to Curative-Intent Surgery or Radiotherapy (proPSMA Study): Clinical Trial Protocol. BJU International, 5, 783-793. https://doi.org/10.1111/bju.14374

[22] Uprimny, C., Kroiss, A.S., Decristoforo, C., Fritz, J., von Guggenberg, E., Kendler, 
D., et al. (2017) (68)Ga-PSMA-11 PET/CT in Primary Staging of Prostate Cancer: PSA and Gleason Score Predict the Intensity of Tracer Accumulation in the Primary Tumour. European Journal of Nuclear Medicine and Molecular Imaging, 6, 941949. https://doi.org/10.1007/s00259-017-3631-6

[23] Turkbey, B., Mena, E., Lindenberg, L., Adler, S., Bednarova, S., Berman, R., et al. (2017) 18F-DCFBC Prostate-Specific Membrane Antigen-Targeted PET/CT Imaging in Localized Prostate Cancer: Correlation with Multiparametric MRI and Histopathology. Clinical Nuclear Medicine, 10, 735-740.

https://doi.org/10.1097/RLU.0000000000001804

[24] Kesch, C., Vinsensia, M., Radtke, J.P., Schlemmer, H.P., Heller, M., Ellert, E., et al. (2017) Intraindividual Comparison of $\wedge$ sup $18^{\wedge}$ F-PSMA-1007 PET/CT, Multiparametric MRI, and Radical Prostatectomy Specimens in Patients with Primary Prostate Cancer: A Retrospective, Proof-of-Concept Study. The Journal of Nuclear Medicine, 11, 1805. https://doi.org/10.2967/jnumed.116.189233

[25] von Klot, C.J., Merseburger, A.S., Boker, A., Schmuck, S., Ross, T.L., Bengel, F.M., et al. (2017) (68)Ga-PSMA PET/CT Imaging Predicting Intraprostatic Tumor Extent, Extracapsular Extension and Seminal Vesicle Invasion Prior to Radical Prostatectomy in Patients with Prostate Cancer. Nuclear Medicine and Molecular Imaging, 4, 314-322. https://doi.org/10.1007/s13139-017-0476-7

[26] Muehlematter, U.J., Burger, I.A., Becker, A.S., Schawkat, K., Hotker, A.M., Reiner, C.S., et al. (2019) Diagnostic Accuracy of Multiparametric MRI versus (68)GaPSMA-11 PET/MRI for Extracapsular Extension and Seminal Vesicle Invasion in Patients with Prostate Cancer. Radiology, 2, 350-358.

https://doi.org/10.1148/radiol.2019190687

[27] Declaration of Helsinki. https://www.wma.net

[28] Medical Research Involving Human Subjects Act.

https://english.ccmo.nl/investigators/legal-framework-for-medical-scientific-researc h/laws/medical-research-involving-human-subjects-act-wmo

[29] Epstein, J.I., Allsbrook, W.C., Amin, M.B., Egevad, L.L. and ISUP Grading Committee (2005) The 2005 International Society of Urological Pathology (ISUP) Consensus Conference on Gleason Grading of Prostatic Carcinoma. The American Journal of Surgical Pathology, 9, 1228-1242. https://doi.org/10.1097/01.pas.0000173646.99337.b1

[30] Lopci, E., Lughezzani, G., Castello, A., Saita, A., Colombo, P., Hurle, R., et al. (2020) Prospective Evaluation of (68)Ga-Labeled Prostate-Specific Membrane Antigen Ligand Positron Emission Tomography/Computed Tomography in Primary Prostate Cancer Diagnosis. European Urology Focus. https://doi.org/10.1016/j.euf.2020.03.004

[31] Zamboglou, C., Drendel, V., Jilg, C.A., Rischke, H.C., Beck, T.I., Schultze-Seemann, W., et al. (2017) Comparison of (68)Ga-HBED-CC PSMA-PET/CT and Multiparametric MRI for Gross Tumour Volume Detection in Patients with Primary Prostate Cancer Based on Slice by Slice Comparison with Histopathology. Theranostics, 1, 228-237. https://doi.org/10.7150/thno.16638

[32] Budaus, L., Leyh-Bannurah, S.R., Salomon, G., Michl, U., Heinzer, H., Huland, H., et al. (2016) Initial Experience of (68)Ga-PSMA PET/CT Imaging in High-Risk Prostate Cancer Patients Prior to Radical Prostatectomy. European Urology, 3, 393 396. https://doi.org/10.1016/j.eururo.2015.06.010

[33] de Rooij, M., Hamoen, E.J.H., Witjes, J.A., Barentsz, J.O. and Rovers, M.M. (2015) Accuracy of Magnetic Resonance Imaging for Local Staging of Prostate Cancer: A Diagnostic Meta-Analysis. European Urology, 2, 233-245. 
https://doi.org/10.1016/j.eururo.2015.07.029

[34] Chen, M., Zhang, Q., Zhang, C., Zhou, Y.H., Zhao, X., Fu, Y., et al. (2020) Comparison of (68)Ga-Prostate-Specific Membrane Antigen (PSMA) Positron Emission Tomography/Computed Tomography (PET/CT) and Multi-Parametric Magnetic Resonance Imaging (MRI) in the Evaluation of Tumor Extension of Primary Prostate Cancer. Translational Andrology and Urology, 2, 382-390.

https://doi.org/10.21037/tau.2020.03.06

[35] Fei, B., Schuster, D.M., Master, V., Akbari, H., Fenster, A. and Nieh, P. (2012) A Molecular Image-Directed, 3D Ultrasound-Guided Biopsy System for the Prostate. Proceedings of SPIE - The International Society for Optical Engineering, Volume 8316, Article ID: 831613. https://doi.org/10.1117/12.912182

[36] Lazzeri, M., Lopci, E., Lughezzani, G., Colombo, P., Casale, P., Hurle, R., et al. (2017) Targeted 11C-Choline PET-CT/TRUS Software Fusion-Guided Prostate Biopsy in Men with Persistently Elevated PSA and Negative mpMRI after Previous Negative Biopsy. European Journal of Hybrid Imaging, 1, 9. https://doi.org/10.1186/s41824-017-0011-1

[37] Zettinig, O., Shah, A., Hennersperger, C., Eiber, M., Kroll, C., Kubler, H., et al. (2015) Multimodal Image-Guided Prostate Fusion Biopsy Based on Automatic Deformable Registration. International Journal of Computer Assisted Radiology and Surgery, 12, 1997-2007. https://doi.org/10.1007/s11548-015-1233-y 